\title{
DETERMINATION OF CONDITIONS FOR NANOPOROUS STRUCTURE FORMATION IN A METALLIC MATERIAL BY PULSE-PERIODIC LASER ACTION
}

\author{
S.P. Murzin, A.I. Safin, A.A. Shimanov, M.V. Blokhin, S.A. Afanasiev \\ Samara National Research University, Samara, Russia
}

\begin{abstract}
Determination of spectrums of samples responses on external vibroexcitation via pulse-periodic laser action by $\mathrm{CO}_{2}$ laser ROFIN DC 010 and registration of their wave forms have been performed. While analysing the samples' responses using pulse-periodical laser action, it has been found that vibration rate increases in the case of frequencies, which are divisible by the frequency of initial oscillation, during the amplitude decrease and the frequency increase. The dependence of heat rate on the nanoporous structure formation in the absence of the metallic material melting has been researched. While heating the sample, its temperature was highest at its centre. An increase of the laser action time led to a rise in the temperature of the sample's centre, causing the rate of pore formation has increased. Since the diffusion coefficient is related to the temperature through an exponential law, the temperature rise is an effective way to increase rate of the process. However, non-permanent elastic deformation, which is caused by high-powered external action, is a necessary condition for developing a generic thermodynamic moving force, which increases the rate of nanoporous structure formation in metallic material.
\end{abstract}

Keywords: formation, nanoporous structure, laser action, metallic material, frequency, vibration rate, temperature.

Citation: Murzin SP, Safin AI, Shimanov AA, Blokhin MV, Afanasiev SA. Determination of conditions for nanoporous structure formation in a metallic material by pulse-periodic laser action. CEUR Workshop Proceedings, 2016; 1638: 89-94. DOI: 10.18287/1613-0073-2016-1638-89-94

\section{Introduction}

The use of pulse-periodic laser action is a progressive way of forming nanoporous layers on the surface of metallic materials. Conditions for formation of such layers in two-component $\mathrm{Cu}-\mathrm{Zn}$ alloy brass L62 with a $60.5-63.5 \%$ coppercontent have been defined in printed works [1-3]. It is stated that as a result of the laser action, single nanopores, as well as ones that form branched channels, appear in the near-surface 
layer and on the surface. Both are uniformly distributed on the area $[4,5]$. Such structures develop via appearance of vacant sites and their coagulation is a result of $\mathrm{Zn}$ sublimation from the material surface, inducing a concentration gradient and diffusion of this component with higher vapour tension at the surface [6-8]. Non-permanent local deformation, caused by high-powered external action, is a condition for intensification of mass transfer in the solid phase of metallic materials [9-11]. At that, laser action with pulse-periodic radiation allows a persistent stress condition to develop on the surface of the samples $[12,13]$. In order to redistribute the power density in the cross-sectional area of the laser beam, the use of a diffractive optical element - focusator of laser radiation, is expedient [14-17]. The aim of this work is to define the conditions for formation of nanoporous structure in metallic materials via pulseperiodic laser action.

\section{Pulse-periodic laser action on the metallic material}

Samples of $\mathrm{Cu}-\mathrm{Zn}$ alloy brass L62 with dimensions of 30x20x0.05 mm have been researched. Spectra of the samples' responses to external vibroexcitation via pulseperiodic laser action have been measured and their wave forms have been defined. Power action has been performed by a ROFIN DC $010 \mathrm{CO}_{2}$ laser with adjustable output power range of 100-1000 W. A three-component scanning laser vibrationmeasuring instrument Polytec ${ }^{\circledR}$ PSV-400-3D was used to measure the vibration rate. The instrument has three scanning heads, each of them fitted with an interferometer and video camera, as well as a PC and three data management controllers. Data management has been synchronized by PSV 3D software, the user interface is used for visualization of dynamic processes in the form of animated 3D oscillation distributions in the investigated frequency range. Pulse-periodic laser action has been performed with frequencies of 100,500 , and $5000 \mathrm{~Hz}$. After the analysis of the samples' responses on the described external vibroexcitation, it has been found that vibration rate increases in case of frequencies, which are divisible by the frequency of initial oscillation, during the amplitude decrease and the frequency increase. Fig. 1 shows images of the sample, which have been re-established with the use of PSV Presentation software. The oscillation shape corresponds to a frequency of $500 \mathrm{~Hz}$. It has been defined that maximum vibration rate occurs on the periphery of the sample; however, the intensity of the pore formation is much lower here than in centre.

A Mikron M7604F thermovision camera was used to research the heating of the samples via laser action. Fig. 2 shows the temperature field of the sample under pulseperiodic laser action. The highest temperature of the sample was in its centre. An increase of the laser action time led to a rise in temperature of the sample's centre area, du to this, an intensity of pore formation increased. The dependence of the heating on the nanopores structure formation in the absence of the metallic material melting has been researched. Inasmuch as the diffusion coefficient is related to the tem- 
perature of an exponential law, the temperature rise is an effective way to increase the atoms mobility. However, non-permanent elastic deformation, which is caused by high-powered external action, is a necessary condition for development of generic thermodynamical moving force, which provides an intensification of the nanoporous structure formation in a metallic material.

\section{Research of the material's fine structure}

Fine structure of the material has been researched with the use of a scanning electron microscope, VEGA $\$ SB, Tescan. It was found that the laser action creates nanoporous structures in near-surface layer. Channel type nanopores $\sim 100 \mathrm{~nm}$ wide, which organize nanoporous network, mainly appear. Dimensions and shapes of the nanopores, equally distributed on the surface of subgrains, are relatively stable.
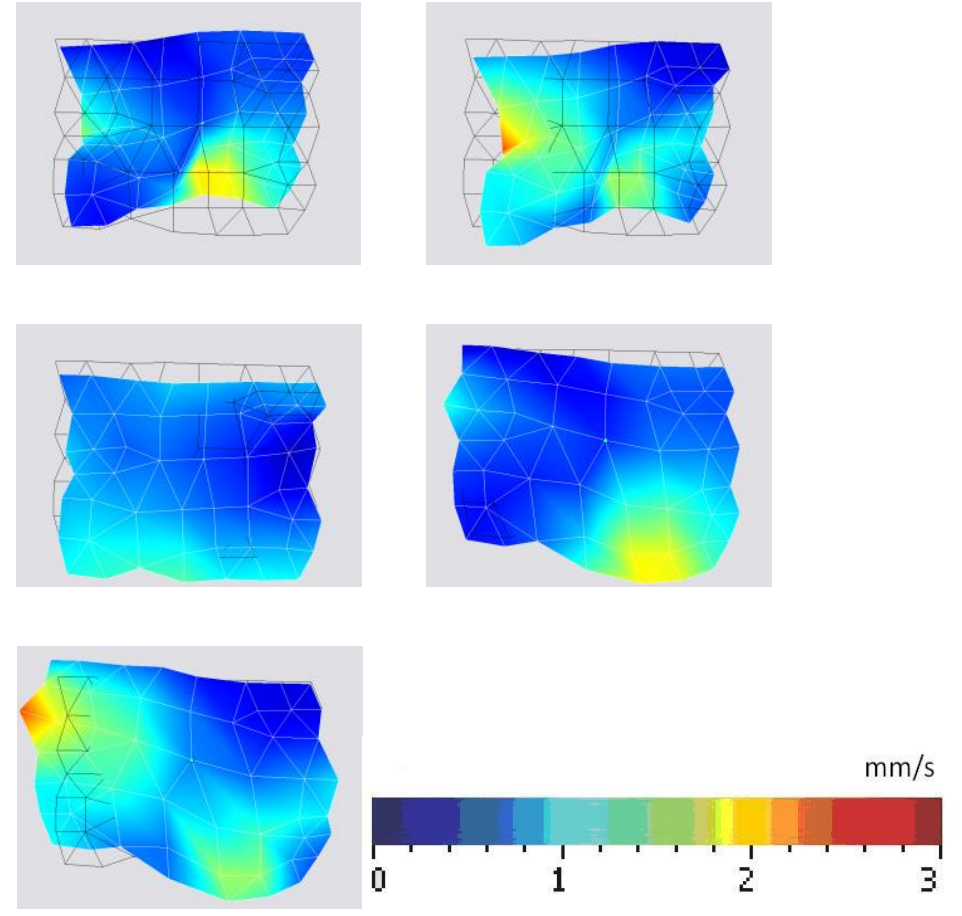

Fig. 1. Images of the sample, which have been re-established with the use of PSV Presentation software and which correspond to the frequency of $500 \mathrm{~Hz}$; time step is $0.2 \mathrm{~ms}$ 


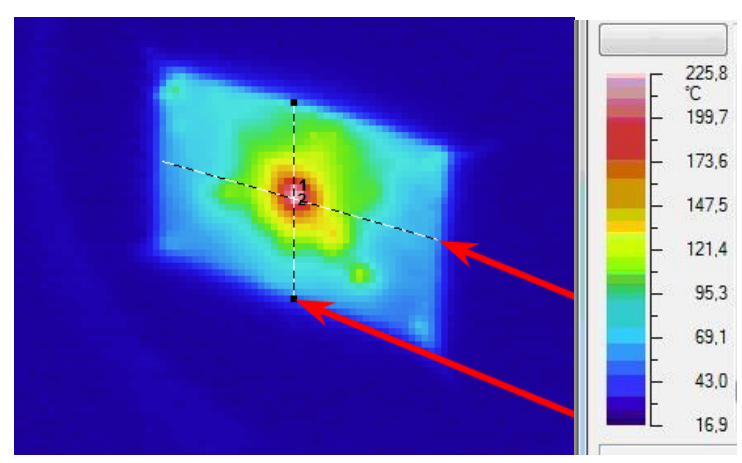

Fig. 2. Temperature field of the sample during its pulse-periodic laser action

Fig. 3 shows image of characteristic nanopore up to $100 \mathrm{~nm}$ wide, which organize into a nanoporous network in the metallic material during its pulse-periodic laser action. The main mechanism of nanoporous structure formation is the sublimation of the component with higher vapour tension; in this case it is $\mathrm{Zn}$. A concentration gradient is set up in the material and this component sublimates with the surface to the extent to which diffusion of it to the surface from the inner layers is provided. In time, the thickness of the $\mathrm{Zn}$ depleted layer increases and the diffusion becomes a limiting factor of the sublimation process. Non-permanent local deformation, caused by highpowered external action, is a condition for intensification of mass transfer inthesolid phase of metallic materials. Inasmuch as the diffusion coefficient is related to the temperature of an exponential law, the temperature rise is an effective way to increase the intensity of the process.

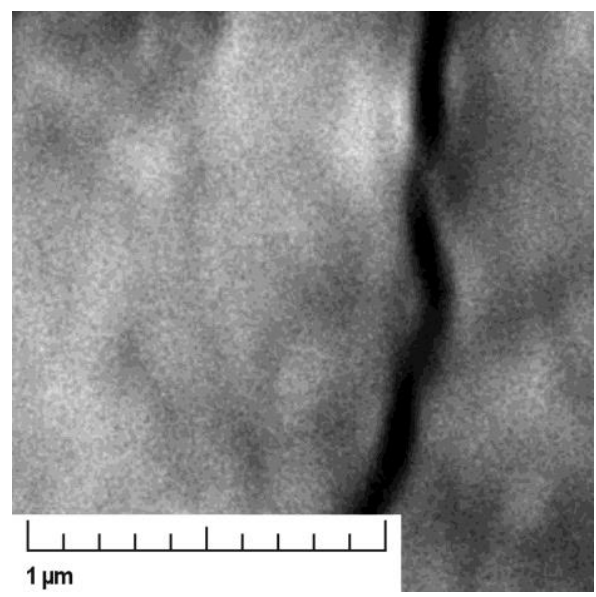

Fig. 3. Image of a $\sim 100 \mathrm{~nm}$ wide nanopore formed as a result of laser action on the sample's metallic surface 


\section{Conclusion}

In analysing of the samples' responses to pulse-periodical laser action, it has been found that the vibration rate increases in case of frequencies, which are divisible by the frequency of initial oscillation, during the amplitude decrease and the frequency increase. While heating the sample, its temperature was highest at its centre. An increase of the laser action time led to a rise in temperature of the sample's centre area, due to this, an intensity of pore formation increased. Non-permanent local deformation, caused by high-powered external action, is a condition for intensification of mass transfer in the solid phase of metallic materials. Inasmuch as the diffusion coefficient is related to the temperature of an exponential law, the temperature rise is an effective way to increase the intensity of the process. The further research will be connected to the definition of self-resonant frequencies and shapes of the oscillations.

\section{References}

1. Kazanskiy NL, Murzin SP, Osetrov YeL, Tregub VI. Synthesis of nanoporous structures in metallic materials under laser action. Opt. Laser Eng., 2011; 49(11): 1264-1267. DOI: 10.1016/j.optlaseng.2011.07.001.

2. Murzin SP. Exposure to laser radiation for creation of metal materials nanoporous structures. Opt. Laser Technol., 2013; 48: 509-512.

3. Murzin SP. The research of intensification's expedients for nanoporous structures formation in metal materials by the selective laser sublimation of alloy's components. Computer Optics, 2011; 35(2): 175-179.

4. Murzin SP. Formation of nanoporous structures in metallic materials by pulse-periodic laser treatment. Opt. Laser Technol., 2015; 72: 48-52.

5. Murzin SP. Determination of conditions for the laser-induced intensification of of mass transfer processes in the solid phase of metallic materials. Computer Optics, 2015; 39(3): 392-396.

6. Murzin SP., Tregub VI, Melnikov AA, Tregub NV. Application of radiation focusators for creation of nanoporous metal materials with high specific surface area by laser action. Computer Optics, 2013; 37(2): 226-232.

7. Murzin SP. Synthesis of metal materials nanoporous structures with cyclic elasto-plastic deformation under laser treatment using radiation focusators. Computer Optics, 2014; 38(2): 249-255.

8. Murzin SP. Method of composite nanomaterials synthesis under metal/oxide pulseperiodic laser treatment. Computer Optics, 2014; 38(3): 469-475.

9. Gertsriken DS, Ignatenko AI, Mazanko VF, Mironova OA, Fal'chenko YuV, Kharchenko GK. Determining the duration of mass transfer and the temperature of metal subjected to pulsed deformation. The Physics of Metals and Metallography, 2005; 99(2): 187-193.

10. Pogorelov AE, Ryaboshapka KP, Zhuravlyov AF. Mass transfer mechanism in real crystals by pulsed laser irradiation. Journal of Applied Physics, 2002; 92(10): 5766-5771.

11. Gercriken DS, Mazanko VF, Tyshkevich VM, Fal'chenko VM. Mass transfer in external influence conditions, iss. 3, corr., RIO IMF, 2001. 443p. [in Russian]. 
12. Murzin SP, Shakhmatov EV, Igolkin AA, Musaakhunova LF. A study of vibration characteristics and determination of the conditions of nanopores formation in metallic materials during laser action. Procedia Engineering, 2015; 106: 266-271.

13. Murzin SP, Kryuchkov AN. Influence of conditions of the samples fixation on the intensity of the nanoporous structure formation in the metallic material by laser action with thermocycling. Procedia Engineering, 2015; 106: 272-276.

14. Golovashkin DL, Kasanskiy NL. Solving diffractive optics problems using graphics processing units. Optical Memory and Neural Networks (Information Optics), 2011; 20(2): 85-89. DOI: 10.3103/S1060992X11020019.

15. Doskolovich LL, Kazansky NL, Kharitonov SI, Soifer VA. A method of designing diffractive optical elements focusing into plane areas. Journal of Modern Optics, 1996; 43(7): 1423-1433. DOI: 10.1080/09500349608232815.

16. Kazanskiy NL. Research \& education center of diffractive optics. Proceedings of SPIE The International Society for Optical Engineering, 2012; 8410(84100R). DOI: $10.1117 / 12.923233$.

17. Kazanskiy NL, Kotlyar VV, Soifer VA. Computer-aided design of diffractive optical elements. Optical Engineering, 1994; 33 (10): 3156-3166. DOI:10.1117/12.178898. 